\title{
Defining a threshold for tacrolimus intra-patient variability associated with late acute cellular rejection in paediatric kidney transplant recipients
}

Introduction: Late acute cellular rejection (LACR) is associated with poorer graft outcomes and non-adherence. Non-adherence to tacrolimus can be indirectly assessed by the intra-patient variability (IPV) of tacrolimus trough levels. The threshold of IPV associated with rejection is not known.

Methods: We conducted a case-control study comparing 25 patients with biopsy proven LACR against 25 stable controls matched for age group, primary diagnosis and time post-transplant. IPV was calculated using coefficient of variance (CV) and mean absolute deviation (MAD) using tacrolimus levels in the preceeding 12 months. We also assessed the percentage time for tacrolimus levels $<4 \mathrm{ug} / \mathrm{L}(\mathrm{Tac}<4)$ and the concentration/weight adjusted dose (C/D) ratio as a proxy marker of tacrolimus metaboliser status.

Results: LACR patients had higher CV (median, IQR: $44 \%, 36-61 \% \vee 24 \%, 19-35 \%, p<0.0001)$ and higher MAD (33\%, 25-48\% v 19\%, 15-26\%). MAD was less affected by outlying tacrolimus results. Receiver operating curve analysis of MAD resulted in a sensitivity of $76 \%$ and specificity of $76 \%$ at a threshold of 26\% (AUC 0.85, p<0.05). LACR patients had more $\mathrm{Tac}<4$ (50\% v 26\%, p<0.05). There was no difference in $C / D$ suggesting that good IPV can be maintained in fast metabolisers. Patients with LACR had significantly increased creatinine at 12-month follow-up despite treatment ( 108 v 5 umol/L increase from baseline) and four patients lost their allograft.

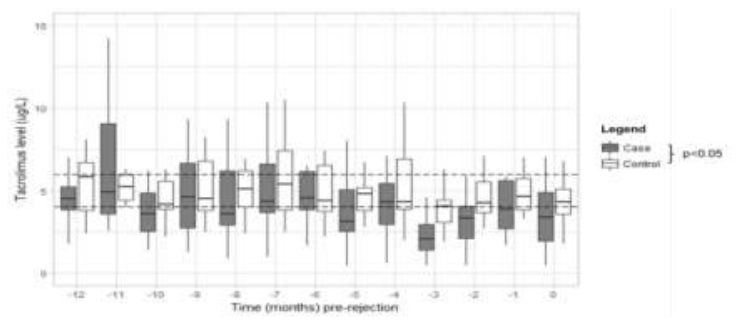

Figure 1: Tacrolimus levels 12 months prior to rejection.

Conclusion: Monitoring of tacrolimus IPV using MAD may be a clinical marker for LACR. A threshold IPV of $26 \%$ can potentially be used as a therapeutic target pending further validation studies.

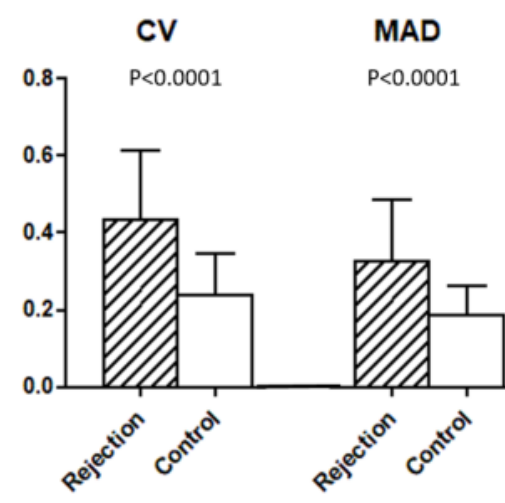

(A)

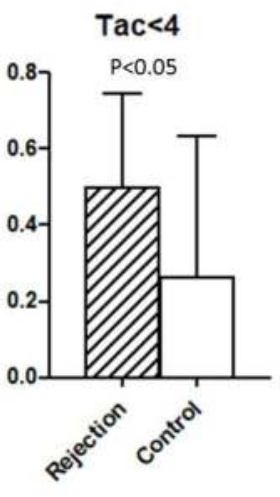

(B)

Figure 2: Tacrolimus IPV (A) and Tac $<4$ (B) for the 12 months preceding LACR. IPV was calculated as coefficient of variance (CV) and mean absolute deviation (MAD). Formulae for calculations provided in the methods.
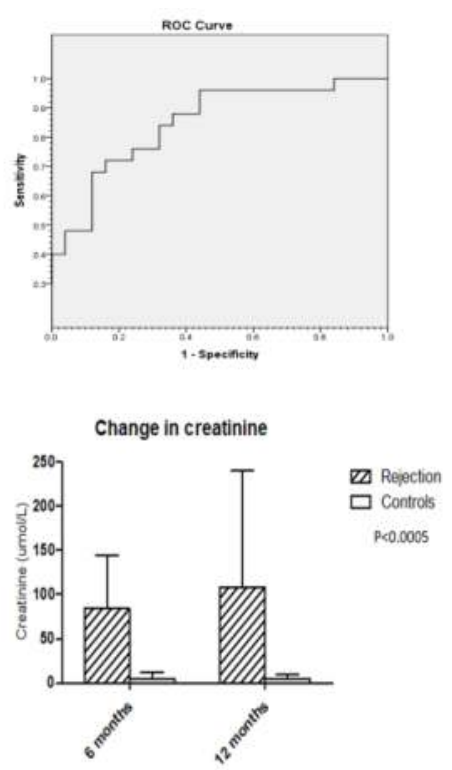

Figure 3: ROC analysis comparing MAD in rejection $v$ controls, AUC $0.85(p<0.05)$.

Figure 4: Follow-up creatinine at 6 and 12 months following treatment for LACR. Changes were taken from baseline and not the creatinine at the time of biopsy. 